\title{
Halitosis amongst students in tertiary institutions in Lagos state
}

\author{
*Joda AE, Olukoju OO
}

Department of Clinical Pharmacy and Bio-pharmacy, Faculty of Pharmacy, University of Lagos

\begin{abstract}
Background: Halitosis is defined as a noticeable unpleasant odor from the mouth. It is a medico-social problem that affects a significant number of people around the world. Research reveals that nearly $50 \%$ of the adult population has halitosis.

Objectives: To determine level of awareness of halitosis and prevalence of the condition amongst students in tertiary institutions as a baseline survey.

Methods: For this project, 100 students from three tertiary institutions in Lagos state were chosen: University of Lagos, Lagos State University, Ojo campus and Yaba College of Technology. A semi-structured questionnaire and practical testing/ diagnostic tool were utilized. Data collected was collated and analyzed using Microsoft Excel 2007 and SPSS statistical software.

Results: Most of the respondents were single and Christian. Level of awareness of halitosis was high. Results showed that $15 \%, 2 \%$ and $22 \%$ from UNILAG, LASU and YCT respectively said they had halitosis. Using the diagnostic tool, $6 \%, 8 \%$ and $2 \%$ respectively were positive for halitosis.

Conclusions: There is high level of awareness of halitosis among the respondents. The prevalence of the disorder is low, however, it is recommended that enlightenment campaigns be mounted in schools to improve level of awareness and treatment seeking.
\end{abstract}

Key words: Halitosis, in-School youth, tertiary institutions, students, Lagos State

African Health Sciences 2012; (4): 473 - 478 http://dx.doi.org/10.4314/ahs.v12i4.12

\section{Introduction}

The term halitosis is derived from the Latin word "halitus", meaning breath and the Greek suffix "osis" meaning condition. Halitosis is defined as a noticeable unpleasant odour that emanates from the mouth which is objectionable to others ${ }^{1}$. It is a medicosocial problem that affects a significant number of people around the world irrespective of race $^{2}$. Halitosis is documented to be the third most frequent reason for seeking dental aid following tooth decay and periodontal disease ${ }^{3,4}$. However, information on the prevalence of halitosis is scarce ${ }^{5}$. Some literature documents that nearly $50 \%$ of the adult population has oral malodour 6 . In a large study involving over 2000 people in Japan, it was reported that $6-23 \%$ of the subjects had halitosis at some time of the day ${ }^{7,8}$. A similar study in the United States revealed

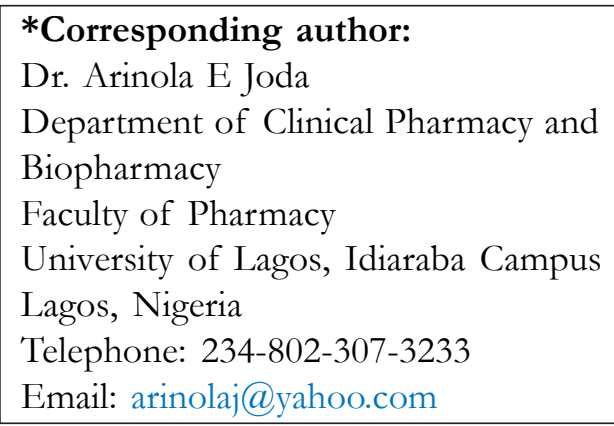

that $24 \%$ of individuals over 60 years old have been told that they had oral malodour?

Many studies on self reported halitosis have stressed that the problem of bad breath is often not self-perceived ${ }^{3}$. In some cases, there is a reduced chance of self detection of oral malodour because the path between the inhaled and exhaled air diverge (while exhaled air travels horizontally, inhaled air travels primarily vertically) $)^{10,11,12}$. The unfortunate fact is that many people do not know that they have a breath problem unless someone directly informs them. Iwakura et al, (1994) ${ }^{3}$ classified people suffering from oral malodour into three groups; Type 1, Selfconscious; Type 2, Conscious by the indication of others, and Type 3, Conscious by presumption from the attitude of others.

Some bad breath, however, are considered to be somewhat normal. One example is "morning mouth" 13 which is as a result of changes in the conditions of the mouth during sleep. During the day, saliva washes decaying food and odours out of the mouth. Less saliva is produced at night thus the mouth becomes dry and dead cells stick to the tongue and the insides of the cheek. When bacteria living in the mouth use these cells for food, they produce foul odour ${ }^{13}$. 
Possible intrinsic causes of halitosis are smoking, alcohol, bad diet and socio-demographic factors ${ }^{7}$. Halitosis also results from poor oral hygiene and other conditions linked to oral cavity or dental problems like periodontitis (inflammation of the gums and ligaments supporting the teeth) and gingivitis ${ }^{6,7}$. Research shows that the primary cause of halitosis is the presence of volatile sulfur compounds (VSCs) in the mouth or in exhaled air . This occurs when a tooth cavity or periodontal (gum) disease allows bacterial infections to thrive in the mouth which then digest oral proteins producing the volatile sulfur compounds as waste products ${ }^{5,14,15,16}$. These VSCs include hydrogen sulfide, dimethyl sulfide and methyl mercaptan. The principal bacteria that have been implicated include Fusobacterium nucleatum, Prevotella intermedia and Tannerella forsythensis ${ }^{14,15}$. The VSCs produce different types of oral malodour and to varying degrees ${ }^{11,12}$. Successful treatment of halitosis depends on the correct diagnosis of the type of halitosis and is categorized into five approaches enabling precise sequential treatment protocol ${ }^{17,18}$.

TN-1 is the basic treatment indicated for all types of halitosis. It includes tongue cleaning and mouth rinsing. TN-2 is strictly cause-related therapy and its aim is to control all oral diseases and all intraoral malodour-causing factors. Patients diagnosed with genuine physiological halitosis are provided with TN-1, whereas patients with genuine pathological intra-oral and extra-oral halitosis are given TN-1 and TN-2 to improve their oral health. Patients with pathological extra-oral halitosis are referred to the appropriate medical practitioner (which is $\mathrm{TN}-3$ ) for management of the systemic condition that is responsible for the oral malodour. TN-4 and TN-5 are additional management strategies for people with psychological halitosis.

The specific management of halitosis could involve the use of drugs and/or herbal remedies ${ }^{19,20}$. Treatment modalities include mechanical reduction of intra-oral nutrients and microorganisms by tongue cleaning, tooth brushing, etc., chemical reduction of oral microbial load using mouth washes, rinses and oxidizing lozenges, rendering malodourous gases nonvolatile and masking the malodour_with mouth sprays, lozenges and chewing gums containing volatiles with pleasant odour.

This study set out to determine the level of awareness of halitosis and the prevalence of the condition amongst students in tertiary institutions as a baseline survey for future research.

\section{Methods}

For this project, three tertiary institutions in Lagos state were randomly chosen from the list of tertiary educational institutions in Lagos State obtained from the State Ministry of Education. These were the University of Lagos, Akoka (Unilag), Lagos State University, Ojo campus (LASU) and Yaba College of Technology (YCT). 100 students from these institutions were conveniently sampled. In each institution, students were approached and the objectives of the survey explained to them at spots were they usually gather (classroom areas, halls of residence and parks) until the sample size is reached. A semi-structured questionnaire to find out demographic background and opinions of the students on the research questions was administered. In addition, practical testing/diagnosis of halitosis was carried out on consenting respondents using a modified tongue scraping test ${ }^{21}$. Tongue scrapping was carried out using a clean, unused toothbrush for each student (instead of a spoon) and the scrapings obtained smelled in order to determine the presence of foul odour. Ninety-six (96) students agreed to practical testing. Data collected was collated and analyzed using the Microsoft Excel 2007 and epiinfo statistical software. Results are presented in form of frequency tables and charts and statistical analysis done using chi square determinations.

\section{Results}

Demographic profile of the respondents showed that $51 \%$ were males with most of them in the age range of between 20 to 24 years of age. Most of the respondents are single and were in the second year of study. The difference in demographic details amongst the schools was statistically significant at 95\% confidence intervals (table 1).

About $92 \%$ of the respondents are aware of halitosis. All students from LASU were aware (figure 1). The difference in this result was statistically significant at 955 confidence internval (critical value - 7.82; degrees of freedom - 6; calculated value 16.66) 
Table 1: Demographic information of respondents

\begin{tabular}{llllll}
\hline Item & Variables & UNILAG $\%$ & LASU $\%$ & YCT \% & Total (\%) \\
\hline Gender & Male & 47 & 40 & 66 & $153(51.0)$ \\
& Female & 42 & 51 & 31 & $124(41.3)$ \\
& Blank & 11 & 9 & 3 & $23(7.7)$
\end{tabular}

Chi sqaure (statistically sig. diff.) critical value $=9.49$ (d.f. 4 ); calculated value $=16.47$

Age (years)

$\begin{array}{lllrc}<19 & 23 & 11 & 24 & 58(19.3) \\ 20-24 & 57 & 66 & 40 & 163(54.3) \\ 25-29 & 9 & 14 & 24 & 47(15.7) \\ 30-34 & 3 & 1 & 6 & 10(3.3) \\ >35 & 1 & 1 & 2 & 4(1.3) \\ \text { Blank } & 7 & 7 & 4 & 18(6.0)\end{array}$

Chi sqaure (Statistically sig. diff.) critical value $=18.31$ (d.f. 10 ); calculated value $=24.58$

Marital status

$\begin{array}{llcll}\text { Married } & 6 & 5 & 19 & 30(10.0) \\ \text { Single } & 86 & 92 & 67 & 245(81.7) \\ \text { Others } & 0 & 0 & 2 & 2(0.7) \\ \text { Blank } & 8 & 3 & 12 & 23(7.7)\end{array}$

Chi sqaure (Statistically sig. diff.) critical value $=12.59$ (d.f. 6); calculated value $=25.65$

\begin{tabular}{lccccr}
\hline Level & 100 & 0 & 0 & 50 & $50(16.7)$ \\
& 200 & 37 & 33 & 30 & $100(33.3)$ \\
& 300 & 23 & 40 & 14 & $77(25.7)$ \\
& 400 & 20 & 13 & 6 & $39(13.0)$ \\
& 500 & 20 & 14 & 0 & $34(11.3)$
\end{tabular}

Chi sqaure (Statistically sig. diff.) critical value $=15.51$ (d.f. 8); calculated value $=140.43$

\begin{tabular}{clcccc}
\hline Religion & Christianity & 75 & 75 & 71 & $221(73.7)$ \\
& Islam & 14 & 17 & 16 & $47(15.7)$ \\
& Others & 1 & 0 & 3 & $4(1.3)$ \\
& Blank & 10 & 8 & 10 & $28(9.3)$
\end{tabular}

Chi sqaure (No statistically sig. diff.) critical value $=12.59$ (d.f. 6); calculated value $=4.24$

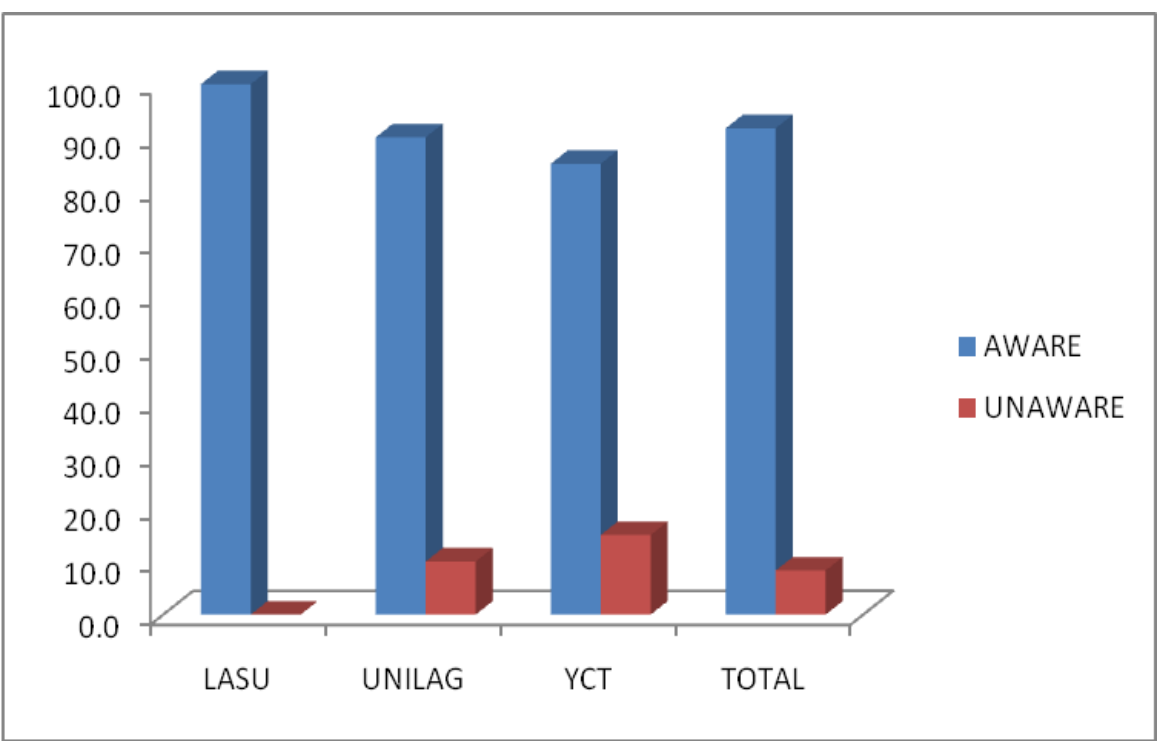

Figure 1: Awareness of halitosis amongst respondents 
Most of the respondents know that halitosis is not contagious while they erroneously believe that the microorganisms implicated in halitosis are viruses (table 2). The difference in the results is statistically significant at $95 \%$ confidence interval.

Table 2: Respondents halitosis knowledge and experience

\begin{tabular}{llllll}
\hline Item & Variables & \multicolumn{2}{l}{ UNILAG \% LASU \% } & YCT \% & Total (\%) \\
\hline Halitosis Contagious? & Yes & 8.0 & 5.0 & 27.0 & $40(13.3)$ \\
& No & 87.0 & 90.0 & 69.0 & $246(82.0)$ \\
& Others & 2.0 & 3.0 & 2.0 & $7(2.3)$ \\
& Blank & 3.0 & 2.0 & 2.0 & $7(2.3)$
\end{tabular}

Chi sqaure (statistically sig. diff.). critical value $=12.59$ (d.f. 6); calculated value $=25.07$

\begin{tabular}{lllccl}
\hline Cause of Halitosis & Bacteria & 0.0 & 5.0 & 6.0 & $11.0(3.7)$ \\
& Fungi & 16.0 & 11.0 & 15.0 & $42.0(14.0)$ \\
& Viruses & 73.0 & 80.0 & 76.0 & $229.0(76.3)$ \\
& Others & 11.0 & 4.0 & 3.0 & $18.0(6.0)$
\end{tabular}

Chi sqaure (statistically sig. diff.). critical value $=12.59$ (d.f. 6); calculated value $=13.28$

$\begin{array}{ccccc}\text { Do you have bad breath? Yes } & 15.0 & 2.0 & 22.0 & 39.0(13.0) \\ \text { No } & 77.0 & 83.0 & 47.0 & 207.0(69.0) \\ \text { Not sure } & 4.0 & 8.0 & 13.0 & 25.0(8.3) \\ \text { Blank } & 4.0 & 7.0 & 18.0 & 29.0(9.7)\end{array}$

Chi sqaure(statistically sig. diff.). critical value $=12.59$ (d.f. 6 ); calculated value $=42.75$

\begin{tabular}{llllll}
\hline Intensity of bad breath & Very intense & $0(0.0)$ & $2(20.0)$ & $3(8.6)$ & $5(7.8)$ \\
& Weak & $2(10.5)$ & $4(40.0)$ & $11(31.4)$ & $17(26.6)$ \\
& Average & $5(26.3)$ & $2(20.0)$ & $6(17.1)$ & $13(20.3)$ \\
& Others & $5(26.4)$ & $0(0.0)$ & $7(20.0)$ & $12(18.8)$ \\
& Blank & $7(36.8)$ & $2(20.0)$ & $8(22.9)$ & $17(26.6)$ \\
& Total & $19(100.0)$ & $10(100.0)$ & $35(100.0)$ & $64(100.0)$ \\
Did you consult a health & & & & \\
care provider? & Yes & $4(21.1)$ & $1(10.0)$ & $16(45.7)$ & $21(32.8)$ \\
& No & $9(47.4)$ & $8(80.0)$ & $15(42.9)$ & $32(50.0)$ \\
& Others & $6(31.6)$ & $1(10.0)$ & $4(11.4)$ & $11(17.2)$ \\
& Total & $19(29.7)$ & $10(15.6)$ & $35(54.7)$ & $64(100.0)$
\end{tabular}

Chi sqaure (statistically sig. diff.). critical value $=9.49$ (d.f. 4 ); calculated value $=16.47$

Of the students surveyed in the three institutions, $15 \%, 2 \%$ and $22 \%$ from Unilag, YCT and LASU respectively said they had halitosis. Some students were not sure if they had bad breath or not. The difference in the result obtained was again statistically significant at 95\% confidence interval. About a quarter of students who believe they have halitosis indicate that it is weak to average (table 2).

Only 21 students who indicated they have bad breath actually sought medical intervention for the perceived bad breath (table 2). The difference in the result obtained was again statistically significant at 95\% confidence interval.

About a third of the respondents consented to the practical testing for halitosis $(36,29$ and 31 students from UNILAG, LASU and YCT respectively. Non-consenting respondents were classified as inconclusive or not tested results). Using 476 this diagnostic tool, $6.3 \%, 8.3 \%$ and $2.1 \%$ from UNILAG, LASU and YCT respectively of those that consented to the test were observed to have bad breath. This is equivalent of $6 \%, 8 \%$ and $2 \%$ of the total number of respondents (figure 2). The differences in results are, however, not statistically significant. 


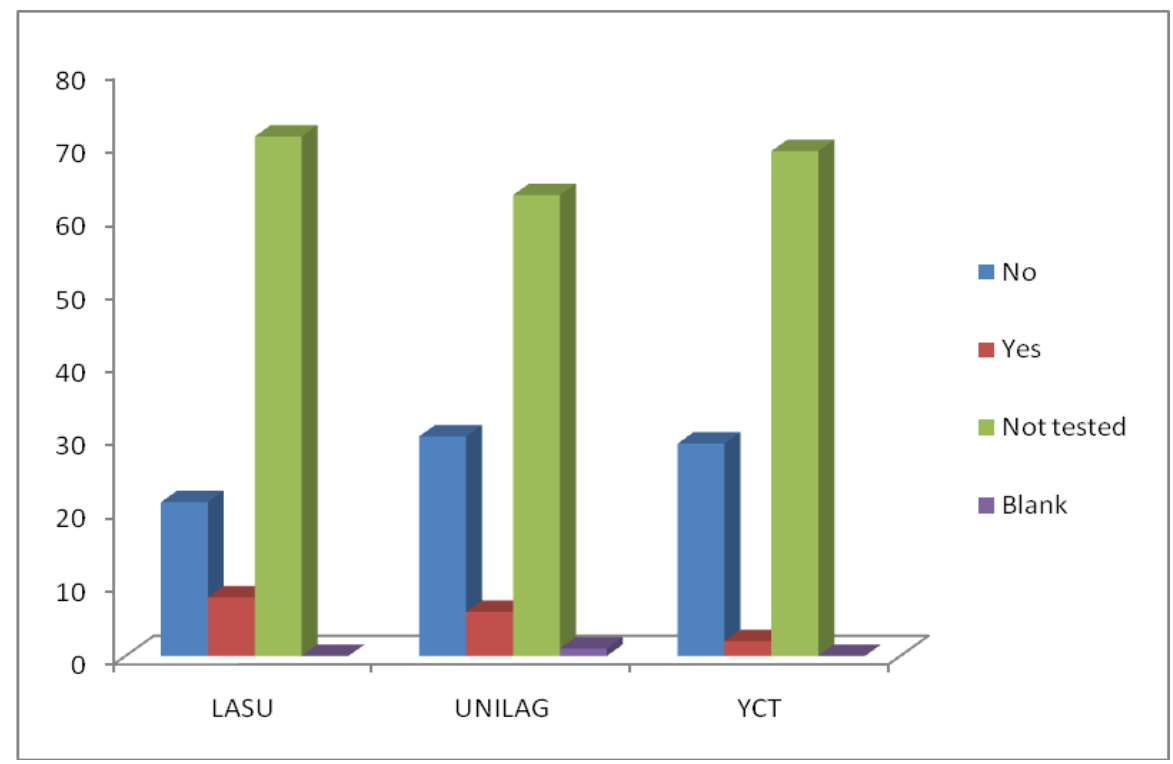

(No statistically significant difference exists)

\section{Figure 2: Halitosis practical demonstration}

\section{Discussion}

The result shows that many students are aware of halitosis as bad breath. From the results the respondents from LASU all had good awareness of halitosis as they could accurately define the term and are all aware that one can have halitosis and not know. Most of the respondents however believe that viruses are more implicated in causing halitosis than bacteria while literature has more bacterial involvement ${ }^{22,23}$

The prevalence of self-perception of oral malodour amongst the respondents was just 13\% unlike a survey carried out amongst patients in a group of Thai dental patients who reported over $60 \%$ prevalence ${ }^{24}$ and a Kuwaiti survey which reported $23 \%{ }^{25}$. However, the number tested positive did not agree with the number who indicated that they know they have the condition confirming Iwakura's argument that cases of halitosis are not self-perceived ${ }^{3}$. There is no statistically significant difference between gender and the selfperceived odour status of the respondents. Research has it that prevalence and incidence ratios between the males and the females are the same though women tend to seek treatment more often than men $^{26}$. The prevalence ratio for halitosis obtained in this study is low using the practical testing tool with only $6 \%, 8 \%$ and $2 \%$ of the respondents having a positive response though many inconclusive results were recorded as only about a third of the respondents agreed to be tested. This result is much lower than

African Health Sciences Vol 12 Issue 4 December 2012 that documented in surveys in the United States where between 24 to $50 \%$ of the adult population has halitosis ${ }^{6,9}$. It however, follows the range obtained in two Japanese surveys were range of 6 to $23 \%$ were obtained ${ }^{7,8}$. Miyazaki H et al. (1999) provided an organoleptic scoring scale ranging from Score 0 (absence of odour) to Score 5 (severe malodour). Score 1 represents questionable odour, Score 2 is slight malodour, Score 3 is moderate malodour and Score 4 is strong malodour ${ }^{17}$.

This survey brings to fore the imbalance between knowledge and action in that it is often argued that possession of knowledge does not necessarily mean people will carry out the correct action. Thus there is a need for enlightenment campaigns and continuous messages on halitosis in tertiary institutions nationwide to enable constant exposure to correct information on halitosis, its identification, prevention and management.

\section{Conclusion}

It can be concluded that though there is good awareness of halitosis among students of tertiary institutions, the prevalence of the disorder is significant. It is recommended that enlightenment campaigns be mounted in schools to improve on the level of awareness, prevention practices and treatment seeking for halitosis. It is also recommended that future surveys targeting respondents knowledge of causes of halitosis be carried out. 


\section{References}

1. ADA Council on Scientific Affairs. Oral Malodour: Journal of American Dental Association 2003; 134(2):209-14.

2. Fillipi A \& Muller, N. Real and psychological halitosis- findings diagnoses and outcome of a halitosis clinic. Schweizer Monatsschrift fur Zabnmedizin 2006;16(2):129-135.

3. Iwakura M, Yasuno Y, Shimura M, Sakamoto, S. Clinical Characteristics of Halitosis: differences in two patient groups with primary and secondary complaints of halitosis. Journal of Dental Research, 1994;73:1568-1574

4. Bosy A. Oral malodour: philosophical and practical aspects. Journal of the Canadian Association 1997;63:196-201.

5. Sanz N, Roldan S, \& Herrera D. Fundamentals of Breath Malodour. Journal of Contemporary Dental Practice, 2001;4(2):001-017.

6. Spielman AI, Bivona P, Rifkin BR. Halitosis - A common oral problem. New York State Dental Journal 1996;62(10):36-42.

7. Attia EL, Marshall KG. Halitosis. Canadian Medical Association Journal, 1982;126:1281.

8. Miyazaki H, Sakao S, Katoh Y, Takehara T. Correlation between volatile sulphur compounds and certain oral health measurements in the general population. Journal of Periodontology, 1995;66:679-84.

9. Van Steenberghe D, Rosenberg M. Bad Breath. A multidisciplinary approach Leuven: Leuven University press, 1996.

10. Oho T, Yoshida Y, Shimazaki Y, Yamashita Y, Koga, T. Characteristic of patients complaining of halitosis and the usefulness of gas chromatography for diagnosing halitosis. Oral Surgery, Oral Medicine, Oral Pathology, Oral Radiology \& Endodontics, 2001;91(5):531-4.

11. Al-Ansari JM, Bordai H, Al-Sumait N, AlKhabbaz A K, Al-Shammari K F, Salako N. Factors associated with self-reported halitosis in Kuwaiti patients. Journal of Dentistry, 2006;34:444-449.

12. Nalcaci R. Factors associated with self-reported halitosis (SRH) and perceived taste disturbance (PTD) in elderly. Archives of Gerontology and Geriatrics, 2008;46(3):307-16.

13. Velde S, Quirynen M, Van Hee P, Van Steenberghe, D. Halitosis associated volatiles in breath of healthy subjects. Journal of Chromatography, 2007; 853:54-61.
14. Richter JL. Diagnosis of Treatment of Halitosis. Compendium of Continuing Education in Dentistry, 1996;17(4):370-2, 374-6.

15. Kaizu T, Tsunoda M, Sato H, Sato T. Reduction of bad breath from periodontal patients by dilute hydrogen peroxide solution. Bulletin Tokyo Dental College, 1978;19:209-16.

16. Calil CM, Marcondes FK. Influence of anxiety on the production of oral volatile sulfur compounds. Life Sciences, 2006;79:660-664.

17. Miyazaki H, Arao M, Okamura K, Kawaguchi Y, Toyofuku A, Hoshi K \& Yaegaki K. Tentative classification of halitosis and its treatment needs. Niigata Dental Journal, 1999;32:7-11.

18. Yaegaki K, Coil J M. Examination, Classification, and Treatment of Halitosis; Clinical Perspectives. Journal of Canadian Dental Association, 2000;66:257-61.

19. Weinberg M. Halitosis: the 'bad breath' syndrome. US Pharmacist 2001;26(3):46- 57.

20. Annemiek M W T, van den Broek, Louw Feenstra, Cees de Baat. A review of the current literature on aetiology and measurement methods of halitosis. Journal of Dentistry, 2007;35 (8): 627-635

21. Hartley G, El-Maaytah M, Greenman, J. Tongue microfl ora of subjects with low and high malodour levels. Journal of Dental Research, 1995;74:587.

22. Feller L, Blignaut E. Halitosis: A Review. Journal of the South African Dental Association, 2005;60(1):17-9.

23. Ongole R, Shenoy N. Halitosis: Much beyond oral malodour. Kathmandu University Medical Journal 2010;8 (2)30:269-275

24. Youngnak-Piboonratanakit P, Vachirarojpisan T. Prevalence of Self-Perceived Oral Malodour in a Group of Thai Dental Patients. Journal of Dentistry, Tehran University of Medical Sciences, Tehran, Iran 2010;7(4).

25. Al-Ansari J M, Booda H, Al-Sumait N, AlKhabbaz A K, Al-Shammari K F, Salako N. Factors associated with self-reported halitosis in Kuwaiti patients. Journal of Dentistry, 2006;34(7):444-449

26. Thrane PS, Young A, Jonski G, Rölla G. A new mouthrinse combining zinc and chlorhexidine in low concentrations provides superior efficacy against halitosis compared to existing formulations: a double-blind clinical study. Journal of Clinical Dentistry, 2007;18(3):826.

African Health Sciences Vol 12 Issue 4 December 2012 